\title{
Efficacy and safety of XELOX and FOLFOX6 adjuvant chemotherapy following radical total gastrectomy
}

\author{
SHI CHEN ${ }^{1,2^{*}}$, XINGYU FENG $^{1 *}$, YUANFANG LI $^{1}$, XIUHONG YUAN $^{1}$, \\ ZHIWEI ZHOU ${ }^{1,2}$ and YINGBO CHEN ${ }^{1}$ \\ ${ }^{1}$ Department of Gastropancreatic Surgery; ${ }^{2}$ The State Key Laboratory of Oncology in South China, \\ Sun Yat-Sen University Cancer Center, Guangzhou, Guangdong, P.R. China
}

Received October 20, 2011; Accepted January 3, 2012

DOI: $10.3892 / \mathrm{ol} .2012 .577$

\begin{abstract}
The purpose of this study was to compare the efficacy and safety of capecitabine and oxaliplatin (XELOX) with 5-fluorouracil, folinic acid and oxaliplatin (FOLFOX6) for advanced gastric cancer following total gastrectomy. We performed a retrospective study of 148 gastric cancer patients undergoing total gastrectomy combined with adjuvant chemotherapy from January, 2003 to June, 2009. The follow-up lasted until December, 2010. The Chi-square test and Kaplan-Meier methods were employed to compare the adverse events and prognosis. The total 1-, 3- and 5-year survival rates were 95, 80 and $32 \%$, respectively, and there was no significant difference between the two groups $(\mathrm{P}=0.273)$. Similarly, the total incidence of side effects was similar, but each treatment was associated with unique disturbances. The number of patients developing hand-foot syndrome in the XELOX group was far higher compared to the FOLFOX6 group $(\mathrm{P}=0.000)$. By contrast, more patients in the FOLFOX6 group suffered from nausea $(\mathrm{P}=0.024)$, vomiting $(\mathrm{P}=0.029)$, alopecia $(\mathrm{P}=0.033)$ and peripheral phlebitis $(\mathrm{P}=0.004)$. The total completion rate of the XELOX group was higher compared to the FOLFOX6 group ( $\mathrm{P}=0.015)$. No significant difference was found in the prognosis of patients receiving XELOX therapy or FOLFOX6 therapy following total gastrectomy. XELOX was, however, more tolerable for patients with total gastrectomy.
\end{abstract}

\section{Introduction}

Gastric cancers are the fourth most common malignancy worldwide, and they are the second most lethal (1-3). In China, the majority of cases have already evolved into advanced

Correspondence to: Dr Yingbo Chen, Sun Yat-Sen University Cancer Center, 651 Dongfeng East Road, Guangzhou 510060, P.R. China

E-mail: chenyb@sysucc.org.cn

*Contributed equally

Key words: gastric cancer, radical total gastrectomy, oxaliplatin, capecitabine, 5-fluorouracil gastric cancer upon diagnosis (4). At present, radical gastrectomy has been regarded as the most effective treatment for gastric cancer. Adjuvant chemotherapy has been proven to benefit patients with advanced gastric cancer following total gastrectomy. The INT0116 study (5) indicated that adjuvant chemotherapy increased the survival rate of cancer patients, whereas in the MAGIC study (6), progression-free survival time in the gastrectomy plus chemotherapy group was significantly longer than the gastrectomy-alone group. In addition, the 5-year survival rate of the surgery plus chemotherapy group was significantly longer than the surgery-alone group. Based on these clinical findings, postoperative adjuvant chemotherapy has been recommended in a Class-I guideline of The National Comprehensive Cancer Network (NCCN) for treating advanced gastric cancer.

In 2007, the ACTS trial (7) revealed that patients at stages II and III benefited from oral S-1 (fluoropyrimidine) following radical gastric surgery. However, those requiring total gastrectomy are in the latest stages, beyond the therapeutic window for S-1. The majority of gastric cancer patients, therefore, require radical gastrectomy and adjuvant chemotherapy. However, total gastrectomy destroys the continuity and integrity of the digestive tract, although there are almost 50 reconstruction methods, such as Roux-en-Y esophagojejunostomy (8). Gastrectomy leads to a number of gastric functional losses, including food storage capacity, mechanical mixing and secretion of digestive juices. This surgery negatively affects digestion and nutrient absorption, and inevitably causes a number of complications, including reflux esophagitis, dumping syndrome, malnutrition and Roux-en-Y stasis syndrome (RSS), which significantly reduce the chemotherapy drug tolerance (9-11). Consequently, few studies have compared the safety and efficacy of various postoperative adjuvant chemotherapies for gastric cancer patients following total gastrectomy. Currently, there is no standard adjuvant chemotherapy following surgery. Chemotherapies including XELOX and FOLFOX6 regimens have been widely applied in clinics. However, few studies have directly compared their safety and efficacy. This clinical trial was designed to retrospectively study gastric cancer patients who received total gastrectomy in the Sun Yat-sen University Cancer Center, and to further evaluate the safety and efficacy of postoperative XELOX and FOLFOX6 adjuvant chemotherapies. 
Table I. Clinical pathological data of all the gastrectomy patients prior to the chemotherapy trial.

\begin{tabular}{|c|c|c|c|c|c|}
\hline \multirow[t]{2}{*}{ Clinical pathological data } & \multicolumn{2}{|c|}{ XELOX group ( $n=87$ cases) } & \multicolumn{2}{|c|}{ FOLFOX6 group ( $\mathrm{n}=61$ cases) } & \multirow[b]{2}{*}{ P-value } \\
\hline & Cases & $\%$ & Cases & $\%$ & \\
\hline \multicolumn{6}{|l|}{ Age (years) } \\
\hline Median & \multicolumn{2}{|c|}{53} & \multicolumn{2}{|c|}{55} & \\
\hline Range & \multicolumn{2}{|c|}{$28-69$} & \multicolumn{2}{|c|}{$23-68$} & \\
\hline \multicolumn{6}{|l|}{ Gender } \\
\hline Male & 56 & 64.4 & 37 & 60.7 & \\
\hline Female & 31 & 35.6 & 24 & 39.3 & 0.730 \\
\hline \multicolumn{6}{|l|}{ Tumor location } \\
\hline Gastric cardia & 14 & 16.1 & 14 & 23.0 & \\
\hline Stomach & 57 & 65.5 & 33 & 54.1 & \\
\hline Total stomach & 13 & 14.9 & 10 & 16.4 & \\
\hline Remnant stomach & 3 & 3.4 & 4 & 6.6 & 0.486 \\
\hline \multicolumn{6}{|l|}{ Borrmann type } \\
\hline I & 2 & 2.3 & 5 & 8.2 & \\
\hline II & 24 & 27.6 & 16 & 26.2 & \\
\hline III & 41 & 47.1 & 28 & 45.9 & \\
\hline IV & 20 & 23.0 & 12 & 19.7 & 0.414 \\
\hline \multicolumn{6}{|l|}{ Pathological staging } \\
\hline High differentiation & 2 & 2.3 & 6 & 9.8 & \\
\hline Median differentiation & 12 & 13.8 & 6 & 9.8 & \\
\hline Low differentiation & 63 & 72.4 & 39 & 63.9 & \\
\hline Poor differentiation ${ }^{\mathrm{a}}$ & 10 & 11.5 & 10 & 16.4 & 0.155 \\
\hline \multicolumn{6}{|l|}{ TNM staging $^{\mathrm{b}}$} \\
\hline $\mathrm{I}^{\mathrm{c}}$ & 4 & 4.6 & 1 & 1.6 & \\
\hline II & 13 & 14.9 & 10 & 16.4 & \\
\hline III & 55 & 63.2 & 42 & 68.9 & \\
\hline IV & 15 & 17.2 & 8 & 13.1 & 0.672 \\
\hline
\end{tabular}

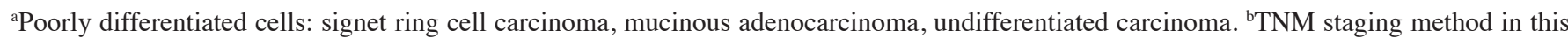
study was in accordance with the standards stated in UICC 2002 TNM Staging Manual for Gastric Cancer (6th edition). ${ }^{\mathrm{C}}$ These TNM stage I patients all had high risk factors such as vessel invasion and poor differentiation stage.

\section{Patients and methods}

\section{Inclusion and exclusion criteria}

Inclusion criteria. Seven inclusion criteria were used for this study: i) histologically proven advanced gastric cancer, total gastrectomy with D2 lymph-node dissection and R0 surgery; ii) patients between 20 and 70 years of age; iii) Eastern Cooperative Oncology Group (ECOG) performance status of 0 or 1 ; iv) no preoperative cancer treatment, such as chemotherapy, immunotherapy or radiotherapy; v) patients receiving chemotherapy by 4 weeks following surgery; vi) no synchronous or metachronous cancers; and vii) adequate organ function with a leukocyte count of at least $4000 / \mathrm{mm}^{3}$ or the lower limit of the normal range, a platelet count of at least $100,000 / \mathrm{mm}^{3}$, a total bilirubin level of not more than $1.5 \mathrm{mg} / \mathrm{dl}(25.7 \mu \mathrm{M})$, aspartate aminotransferase and alanine aminotransferase levels not more than 2.5 times the upper limit of the normal range, and a serum creatinine level not higher than the upper limit of the normal range.
Exclusion criteria. Three exclusion criteria were employed in this study: a) age $>70$ years or $<20$ years; b) hepatic, renal, pulmonary or cardiac dysfunctions; c) severe postoperative complications, such as anastomosis leakage and anastomosis stenosis that may cause malnutrition or make the patients intolerant to postoperative chemotherapy.

Patient characteristics. From January, 2003 to June, 2009, patients who underwent total gastrectomy received adjuvant chemotherapy at the Sun Yat-sen University Cancer Center. Prior to adjuvant chemotherapy, routine blood test results and blood biochemical indices were consistent with the inclusion criteria. No contra-indications against adjuvant chemotherapy were noted. All the patients gave their informed consent prior to the adjuvant chemotherapy. Following surgery, 87 cases received XELOX chemotherapy, and 61 cases underwent FOLFOX6 chemotherapy. The general clinical data from all patients prior to chemotherapy are presented in Table I. No significant differences in pathological characteristics were 
Table II. Rates of all adverse reactions in the FOLFOX6 and XELOX treatment groups.

\begin{tabular}{|c|c|c|c|c|c|}
\hline \multirow[b]{2}{*}{ Symptom } & \multicolumn{2}{|c|}{ XELOX group $(n=87)$} & \multicolumn{2}{|c|}{ FOLFOX6 group $(n=61)$} & \multirow[b]{2}{*}{ P-value } \\
\hline & Cases & $\%$ & Cases & $\%$ & \\
\hline At least one adverse event & 75 & 86.2 & 54 & 88.5 & 0.805 \\
\hline Nausea & 58 & 66.7 & 51 & 83.6 & $0.024^{\mathrm{a}}$ \\
\hline Vomiting & 31 & 35.6 & 33 & 54.1 & $0.029^{\mathrm{a}}$ \\
\hline Anorexia & 64 & 73.6 & 52 & 85.2 & 0.107 \\
\hline Oral mucositis & 5 & 5.7 & 5 & 8.2 & 0.741 \\
\hline Diarrhea & 20 & 23.0 & 19 & 31.1 & 0.343 \\
\hline Alopecia & 2 & 2.3 & 7 & 11.5 & $0.033^{\mathrm{a}}$ \\
\hline Hand-foot syndrome & 53 & 60.9 & 2 & 3.3 & $0.000^{\mathrm{a}}$ \\
\hline Peripheral neurotoxicity & 64 & 73.6 & 52 & 85.2 & 0.107 \\
\hline Fatigue & 24 & 27.6 & 19 & 31.1 & 0.714 \\
\hline Leukocyte/neutropenia & 50 & 57.5 & 40 & 65.6 & 0.393 \\
\hline Hemoglobin decrease & 5 & 5.7 & 2 & 3.3 & 0.700 \\
\hline Thrombocytopenia & 26 & 29.9 & 13 & 21.3 & 0.083 \\
\hline ALT/AST increase & 29 & 33.3 & 24 & 39.3 & 0.489 \\
\hline TBIL increase & 1 & 1.1 & 2 & 3.3 & 0.569 \\
\hline $\mathrm{Cr} / \mathrm{BUN}$ increase & 1 & 1.1 & 2 & 3.3 & 0.569 \\
\hline Allergy & 1 & 1.1 & 1 & 1.6 & 0.999 \\
\hline Peripheral phlebitis & 0 & 0 & 6 & 9.8 & $0.004^{\mathrm{a}}$ \\
\hline
\end{tabular}

ALT, alanine aminotransferase; AST, aspartate aminotransferase; TBIL, total bilirubin; $\mathrm{Cr}$, serum creatinine; BUN, blood urea nitrogen; ${ }^{\mathrm{a}} \mathrm{P}<0.05$.

noted between the two patient groups prior to treatment $(\mathrm{P}>0.05)$.

Chemotherapy regimen. The XELOX regimen consisted of oxaliplatin at $130 \mathrm{mg} / \mathrm{m}^{2}$ (i.v. drip, day 1) combined with capecitabine at $1000 \mathrm{mg} / \mathrm{m}^{2}$ (oral, day 1-14) administered once every three weeks. The median chemotherapy duration was 7 cycles.

The FOLFOX6 regimen consisted of oxaliplatin at $100 \mathrm{mg} /$ $\mathrm{m}^{2}$ (i.v. drip, day 1) with FA at $400 \mathrm{mg} / \mathrm{m}^{2}$ (racemic) or $200 \mathrm{mg}$ / $\mathrm{m}^{2}$ (L-form) plus 5 -FU at $400 \mathrm{mg} / \mathrm{m}^{2}$ bolus plus $2400 \mathrm{mg} / \mathrm{m}^{2}$ as a continuous 46-h infusion once every 2 weeks. The median chemotherapy duration was 8 cycles.

Adverse events. Adverse reactions were graded on a 0-4 scale for acute and subacute toxicity in accordance with the WHO guidelines for anti-cancer drugs.

Follow up. Following treatment, the patients were monitored every 3 months for the first 2 years, then every 6 months thereafter. Telephone calls and letters were used to assess patients who could not make follow-up assessments regularly. Complete data were collected for all 148 patients through to November 2010. The following-up period ranged from 6 months to 65 months (median, 41.5 months).

Statistical methods. The Chi-square test was used to compare categorical variables and the rates of adverse reactions observed in XELOX-treated and FOLFOX6-treated patients. Student's t-tests were used to compare the continuous variables. Univariate survival analysis was performed using Kaplan-Meier methods. Survival curves were compared with the log-rank test. Statistical analyses were performed with SPSS software version 16.0 for Windows (SPSS, Inc., Chicago, IL, USA). $\mathrm{P}<0.05$ was considered to indicate a statistically significant difference.

\section{Results}

Comparison of the adverse effects of XELOX and FOLFOX. Comparison of all toxicities and side effects. No mortalities were reported in either group within 28 days of the last treatment course. In the XELOX group, 75 of 87 patients (86.2\%) had adverse reactions of variable severity, while 54 of the 61 patients treated with FOLFOX $(88.5 \%)$ also reported toxic side effects $(\mathrm{P}=0.805)$. The most commonly observed adverse reactions in the XELOX-treated patients included peripheral neurotoxicity $(73.6 \%)$, anorexia $(73.6 \%)$, nausea $(66.7 \%)$ and hand-foot syndrome (60.9\%). In the FOLFOX6 group, anorexia $(85.2 \%)$, peripheral neurotoxicity $(85.2 \%)$ and nausea $(83.6 \%)$ were the most frequently occurring adverse events. More patients in the XELOX group showed hand-foot syndrome $(60.9 \%)$ than those in the FOLFOX6 group $(3.3 \%)(\mathrm{P}=0.000)$. In the FOLFOX6 group, a significantly greater number of patients reported nausea (83.6 vs. $66.7 \%$ of XELOX-treated patients, $\mathrm{P}=0.024)$, vomiting (54.1 vs. $35.6 \%, \mathrm{P}=0.029$ ), alopecia 
Table III. Rates of grade 3/4 adverse reactions in FOLFOX6 and XELOX treatment groups.

\begin{tabular}{|c|c|c|c|c|c|}
\hline \multirow[b]{2}{*}{ Adverse reaction } & \multicolumn{2}{|c|}{ XELOX group $(\mathrm{n}=87)$} & \multicolumn{2}{|c|}{ FOLFOX6 group $(\mathrm{n}=61)$} & \multirow[b]{2}{*}{ P-value } \\
\hline & Cases & $\%$ & Cases & $\%$ & \\
\hline At least one adverse event & 38 & 43.7 & 30 & 49.2 & 0.615 \\
\hline Nausea & 8 & 9.2 & 7 & 11.5 & 0.783 \\
\hline Vomiting & 4 & 4.6 & 5 & 8.2 & 0.489 \\
\hline Anorexia & 10 & 11.5 & 8 & 13.1 & 0.802 \\
\hline Oral mucositis & 0 & 0 & 0 & 0 & - \\
\hline Diarrhea & 6 & 6.9 & 5 & 8.2 & 0.761 \\
\hline Alopecia & 0 & 0 & 0 & 0 & - \\
\hline Hand-foot syndrome & 8 & 9.2 & 0 & 0 & $0.021^{\mathrm{a}}$ \\
\hline Peripheral neurotoxicity & 13 & 14.9 & 11 & 18.0 & 0.655 \\
\hline Fatigue & 0 & 0 & 0 & 0 & - \\
\hline Leukocyte/neutropenia & 6 & 6.9 & 8 & 13.1 & 0.257 \\
\hline Hemoglobin decrease & 0 & 0 & 0 & 0 & - \\
\hline Thrombocytopenia & 6 & 6.9 & 6 & 9.8 & 0.553 \\
\hline ALT/AST increase & 2 & 2.3 & 2 & 3.3 & 0.999 \\
\hline TBIL increase & 0 & 0 & 0 & 0 & - \\
\hline $\mathrm{Cr} / \mathrm{BUN}$ increase & 0 & 0 & 0 & 0 & - \\
\hline Allergy & 0 & 0 & 0 & 0 & - \\
\hline Peripheral phlebitis & 0 & 0 & 0 & 0 & - \\
\hline
\end{tabular}

${ }^{\mathrm{a}} \mathrm{P}<0.05$. ALT, alanine aminotransferase; AST, aspartate aminotransferase; TBIL, total bilirubin; $\mathrm{Cr}$, serum creatinine; BUN, blood urea nitrogen .

Table IV. Dosage adjustment, chemotherapy delay and chemotherapy discontinuation in XELOX and FOLFOX6 groups.

\begin{tabular}{|c|c|c|c|c|c|}
\hline & \multicolumn{2}{|c|}{ XELOX group $(\mathrm{n}=87)$} & \multicolumn{2}{|c|}{ FOLFOX6 group $(n=61)$} & \multirow[b]{2}{*}{ P-value } \\
\hline & Cases & $\%$ & Cases & $\%$ & \\
\hline Chemotherapy discontinuation & 5 & 5.7 & 5 & 8.2 & 0.741 \\
\hline \multicolumn{6}{|l|}{ Dosage reduction } \\
\hline XELOX & 5 & 5.7 & 4 & 6.6 & 0.999 \\
\hline Capecitabine or 5-FU & 7 & 8.0 & 2 & 3.3 & 0.308 \\
\hline Chemotherapy delay & 9 & 10.3 & 12 & 19.7 & 0.151 \\
\hline
\end{tabular}

(11.5 vs. $2.3 \%, \mathrm{P}=0.033)$ and peripheral phlebitis ( 9.8 vs. $0 \%$, $\mathrm{P}=0.004)$. No significant difference in rates of other side effects was noted (Table II).

Comparison of $3 / 4$ grade adverse reactions between XELOX and FOLFOX6 treatment groups. A total of 38 cases in the XELOX group (43.7\%) presented with grade $3 / 4$ adverse events, a rate similar to the FOLFOX6 group (30 patients, $49.2 \%, \mathrm{P}=0.615$ ). The most commonly observed adverse events in XELOX-treated patients included peripheral neurotoxicity $(14.9 \%)$, anorexia $(11.5 \%)$, nausea $(9.2 \%)$ and hand-foot syndrome (9.2\%). In the FOLFOX6 group, peripheral neurotoxicity (18.0\%), leukocyte/neutropenia (13.1\%), anorexia (13.1\%) and nausea (11.5\%) were the most frequently occurring grade $3 / 4$ side effects. More patients in the XELOX group had hand-foot syndrome than those in the FOLFOX6 group ( 9.2 vs. $0 \%, \mathrm{P}=0.021)$. One case of ascites due to impaired liver func- tion was found in the XELOX group; this patient recovered following discontinuation of chemotherapy and application of liver protection therapy. No statistical differences in other severe adverse reactions were observed (Table III).

Dosage adjustment, chemotherapy delay and chemotherapy discontinuation in XELOX and FOLFOX6 groups. During the course of chemotherapy, 5 patients in the FOLFOX6 group $(8.2 \%)$ discontinued chemotherapy due to severe adverse reactions, and 5 cases in the XELOX group also discontinued therapy (5.7\%). In total, 4 XELOX-treated patients (6.6\%) and 5 FOLFOX6-treated patients (5.7\%) were administered reduced dosages due to severe toxic side effects. Two of these XELOX-treated patients (3.3\%) required reduced capecitabine and 7 FOLFOX6-treated cases (8.0\%) required a reduced 5-FU dosage. A total of 12 XELOX-treated patients (19.7\%) and 
Table V. Patient prognosis in XELOX and FOLFOX6 treatment groups.

\begin{tabular}{lcccc}
\hline \multirow{2}{*}{ Group } & \multicolumn{3}{c}{ Survival rate } & \multirow{2}{*}{ P-value } \\
\cline { 2 - 4 } & 1-year (\%) & 3-year (\%) & 5-year (\%) & \\
\hline XELOX & 72 & 43 & 34 & 0.582 \\
FOLFOX & 68 & 45 & 29 & \\
\hline
\end{tabular}

$\mathrm{P}>0.05$.

9 FOLFOX6-treated patients (10.3\%) delayed chemotherapy due to severe adverse reactions. No significant difference in clinical outcome was observed between the two treatment groups (Table IV).

Comparison of patient prognosis between XELOX and FOLFOX6 groups. The 1-, 3- and 5-year survival rates of 148 patients following radical total gastrectomy were 95, 80 and $32 \%$, respectively. Results comparing patient prognosis between the two treatment groups are shown in Table V and Fig. 1.

\section{Discussion}

Chemotherapy for gastric cancers has shown marked progress in recent years, facilitated by numerous basic and clinical studies focusing on the necessity and efficacy of postoperative chemotherapy to complement surgical treatment (5-7). For those patients with whole gastric cancer, proximal gastric cancer and diffuse gastric cancer, total gastrectomy remains the main curative therapy, particularly for malignant tumors occurring at the gastric-esophageal junction. The majority of clinicians now recommend total gastrectomy for these patients to reduce gastric reflux $(12,13)$. Previous findings indicated that patients requiring total gastrectomy were usually diagnosed with late stage cancer by pathological observations (4), necessitating post-operative chemotherapy.

Total gastrectomy destroys the continuity and integrity of the digestive tract, however, and leads to reflux esophagitis, dumping syndrome, malnutrition and Roux-en-Y stasis syndrome (RSS). These conditions negatively affect post-surgery quality of life and postoperative tolerance to adjuvant chemotherapy. Thus, there has been no consensus on a standard chemotherapy regimen. The most commonly used drugs, including amycin, cisplatin and mitomycin, induced relatively severe gastrointestinal reactions and bone marrow inhibition. The identification of chemotherapy drugs with improved efficacy but lower toxicity is an ongoing quest for all cancer specialists. In this regard, recent findings indicated that the XELOX regimen demonstrated greater safety and better efficacy in advanced gastric cancer $(14,15)$.

A variety of adverse reactions were reported during the two treatment regimens, and no significant difference was observed in either total side effect rates $(\mathrm{P}=0.805)$ or more severe grade $3 / 4$ reactions $(\mathrm{P}=0.615)$. However, no patient died during chemotherapy, indicating that both treatments were relatively safe. This study aimed to compare the XELOX

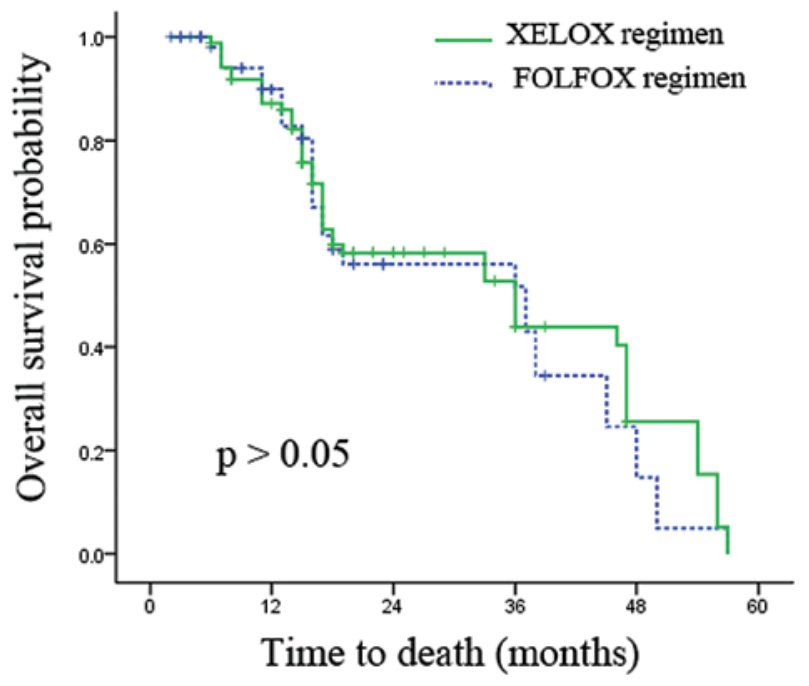

Figure 1. Survival rates following chemotherapy using XELOX or FOLFOX6. There is no statistical significant difference between these two groups. $\mathrm{P}>0.05$.

regimen with FOLFOX6 and highlight its clinical efficacy and tolerability. While overall rates of side effects were similar, the number of patients developing severe digestive tract toxicity, as evidence by nausea and vomiting, was significantly lower than that in the FOLFOX6 group. This is a significant advantage for patients with total gastrectomy, as these patients inevitably present severe digestive tract complications that reduce resistance and tolerability of patients towards chemotherapy. In addition, the XELOX regimen required fewer treatment courses ( 8 in total), and the majority of patients could complete the whole round of chemotherapy. In this trial, 39 XELOXtreated patients $(44.8 \%)$ completed the whole chemotherapy trial, while the FOLFOX6 regimen required 12 courses and, therefore, significantly fewer patients $(24.6 \%)$ completed the trial $(\mathrm{P}=0.015)$. The XELOX regimen was easier to apply due to this higher completion rate.

While more patients completed the XELOX trial, rates of severe side effects were similar. Few patients in the FOLFOX6 group completed the treatment; however, such severe adverse reactions might have been even more common if more FOLFOX6 patients had continued or completed the required 12 courses. Findings of certain studies (16) have revealed that neurolesions were caused by the accumulation of platinum in dorsal root ganglia. De Gramont et al (17) reported that approximately $15 \%$ of patients showed cumulative neurotoxicity when the accumulative amount of XELOX reached $750 \mathrm{mg} / \mathrm{m}^{2}$. Consequently, the incidence of accumulative neurotoxicity depended on two factors: treatment dosage and treatment time course. A longer treatment course inevitably aggravated the neurotoxicity induced by cumulative XELOX. In addition, FOLFOX6 was provided biweekly with a relatively short interval. Frequent hospital visits and adverse effects on eating, drinking and rest forced more patients who were poorly tolerant to stop FOLFOX6 chemotherapy.

In addition, capecitabine was administered orally in the XELOX regimen, which has unique advantages. In contrast to the injection of 5-FU, oral dosing of capecitabine precluded 
peripheral phlebitis. The FOLFOX6 regimen resolved this problem by using a central venous catheter, but this technique is more invasive, inevitably increases the surgical risk, may lead to infection, and interferes with quality of life. Indeed, patients receiving the XELOX regimen did not need to stay in the hospital as long, and, in general, preferred this regimen over FOLFOX6 treatment.

The XELOX regimen also induced many adverse reactions $(18,19)$. First, the incidence of hand-foot syndrome was relatively high, but notably, not severe enough for many patients to stop chemotherapy. Furthermore, hand-foot syndrome may be mitigated by taking certain precautions. Previous findings (20-22) reported that a large dose of vitamin B6 (200 mg/ day, i.v. or $30 \mathrm{mg}$ orally, 3 times a day) reduced the severity of hand-foot syndrome. Hand-foot syndrome is regarded as an inflammatory reaction, probably associated with the overexpression of COX-2. A retrospective analysis indicated that hand-foot syndrome was relieved by taking the COX-2 inhibitor celecoxib. Finally, skin symptoms such as furfur, ulcers and pain were alleviated by topical lanolin cream. Additionally, two XELOX-treated patients developed severe liver dysfunction, and one case presented ascites. Following careful review of the medical history of the two patients, we found that they had a history of hepatitis B, although they were normal at preoperative examination. Oral administration of capecitabine exacerbated the metabolic burden on liver function and enhanced potential damage. Consequently, we highly recommend that patients with a history of liver disease should be treated with caution, even if preoperative tests are normal.

There was no significant difference in safety between XELOX and FOLFOX6 treatments. Five-year survival rates were not significantly different ( 34 vs. $29 \%, \mathrm{P}=0.273$ ), suggesting that the long-term efficacy of XELOX and FOLFOX6 therapies were similar for patients with radical total gastrectomy.

Currently, there is a major research focus on adjuvant chemotherapy following gastric cancer surgery. Numerous cases are diagnosed at an advanced stage, requiring total gastrectomy, and few studies have focused on adjuvant chemotherapy in this patient group (23). In view of the specific functional limitations of patients with total gastrectomy, particularly reduced gastric function and concomitant sensitivity of gastric side effects, we suggest that XELOX should be applied in clinics due to higher patient tolerance, fewer adverse events and superior compliance.

\section{References}

1. Hazard L, O'Connor J and Scaife C: Role of radiation therapy in gastric adenocarcinoma. World J Gastroenterol 12: 1511-1520, 2006.

2. Jemal A, Siegel R, Ward E, Murray T, Xu J and Thun MJ: Cancer statistics. CA Cancer J Clin 57: 43-66, 2007.

3. Crew KD and Neugut AI: Epidemiology of gastric cancer. World J Gastroenterol 12: 354-362, 2006.
4. Zhan YQ, Li W, Sun XW, et al: Long-term results of surgical treatment of stomach cancer: clinical experience of forty years from Sun Yat-sen University Cancer Center. Zhonghua Wai Ke Za Zhi 43: 1109-1113, 2005 (In Chinese).

5. Macdonald JS, Smalley SR, Benedetti J, et al: Chemoradiotherapy after surgery compared with surgery alone for adenocarcinoma of the stomach or gastroesophageal junction. N Engl J Med 345: 725-730, 2001.

6. Cunningham D, Allum WH, Stenning SP, et al: Perioperative chemotherapy versus surgery alone for resectable gastroesophageal cancer. N Engl J Med 355: 11-20, 2006.

7. Sakuramoto S, Sasako M, Yamaguchi T, et al: Adjuvant chemotherapy for gastric cancer with S-1, an oral fluoropyrimidine. N Engl J Med 357: 1810-1820, 2007.

8. Espat NJ and Karpeh M: Reconstruction following total gastrectomy: a review and summary of the randomized prospective clinical trials. Surg Oncol 7: 65-69, 1998.

9. Stael von Holstein C, Walther B, Ibrahimbegovic E and Akesson B: Nutritional status after total and partial gastrectomy with Roux-en-Y reconstruction. Br J Surg 78: 1084-1087, 1991.

10. Gockel I, Pietzka S and Junginger T: Quality of life after subtotal resection and gastrectomy for gastric cancer. Chirurg 76: 250-257, 2005 (In German).

11. Wu LL, Liang H, Zhang RP, Pan Y and Wang BG: Comparative study on four different reconstruction procedures after total gastrectomy. Zhonghua Wei Chang Wai Ke Za Zhi 13: 895-898, 2010 (In Chinese).

12. Yoo CH, Sohn BH, Han WK and Pae WK: Long-term results of proximal and total gastrectomy for adenocarcinoma of the upper third of the stomach. Cancer Res Treat 36: 50-55, 2004.

13. Wei HB, Wei B, Zheng ZH, et al: Comparative study on three types of alimentary reconstruction after total gastrectomy. J Gastrointest Surg 12: 1376-1382, 2008.

14. Park YH, Kim BS, Ryoo BY and Yang SH: A phase II study of capecitabine plus 3-weekly oxaliplatin as first-line therapy for patients with advanced gastric cancer. Br J Cancer 94: 959-963, 2006.

15. Qian J, Qin SK, Mei JF, Chen YX, Shao ZJ and He ZM: Oxaliplatin plus capecitabine as a second line chemotherapy for patients with advanced gastric cancer. Zhonghua Zhong Liu Za Zhi 26: 746-748, 2004 (In Chinese).

16. Luo FR, Wyrick SD and Chaney SG: Comparative neurotoxicity of oxaliplatin, ormaplatin, and their biotransformation products utilizing a rat dorsal root ganglia in vitro explant culture model. Cancer Chemother Pharmacol 44: 29-38, 1999.

17. De Gramont A, Bosset JF, Milan C, et al: Randomized trial comparing monthly low-dose leucovorin and fluorouracil bolus with bimonthly high-dose leucovorin and fluorouracil bolus plus continuous infusion for advanced colorectal cancer: a French intergroup study. J Clin Oncol 15: 808-815, 1997.

18. Lu GC, Fang F and Li DC: Efficacy and toxicity analysis of XELOX and FOLFOX4 regimens as adjuvant chemotherapy for stage III colorectal cancer. Zhonghua Zhong Liu Za Zhi 32: 152-155, 2010 (In Chinese).

19. Dong NN, Wang MY, Zhang Q and Liu ZF: Oxaliplatin combined with capecitabine as first-line chemotherapy for patients with advanced gastric cancer. Ai Zheng 28: 412-415, 2009 (In Chinese).

20. Jucgla A and Sais G: Diagnosis in oncology. Hand-foot syndrome. J Clin Oncol 15: 3164, 1997.

21. Zhang RX, Wu XJ, Lu SX, Pan ZZ, Wan DS and Chen G: The effect of COX-2 inhibitor on capecitabine-induced hand-foot syndrome in patients with stage II/III colorectal cancer: a phase II randomized prospective study. J Cancer Res Clin Oncol 137: 953-7, 2011.

22. Kamil M, Haron M, Yosuff N, Khalid I and Azman N: High frequency of hand foot syndrome with capecitabine. J Coll Physicians Surg Pak 20: 421-422, 2010.

23. Ott K, Lordick F, Blank S and Buchler M: Gastric cancer: surgery in 2011. Langenbecks Arch Surg 396: 743-758, 2011. 